\title{
Oxytocin Makes a Face in Memory Familiar
}

\author{
Ulrike Rimmele, ${ }^{1,2}$ Karin Hediger, ${ }^{1}$ Markus Heinrichs, ${ }^{1}$ and Peter Klaver ${ }^{3}$ \\ ${ }^{1}$ Department of Psychology, Clinical Psychology and Psychobiology, University of Zurich, 8050 Zurich, Switzerland, ${ }^{2}$ Department of Psychology, New York \\ University, New York, New York 10003, and ${ }^{3}$ Magnetic Resonance Center, University Children's Hospital Zurich, University of Zurich, 8032 Zurich, \\ Switzerland
}

Social recognition is the basis of all social interactions. Here, we show that, in humans, the evolutionarily highly conserved neuropeptide oxytocin, after intranasal administration, specifically improves recognition memory for faces, but not for nonsocial stimuli. With increased oxytocin levels, previously presented faces were more correctly assessed as "known," whereas the ability of recollecting faces was unchanged. This pattern speaks for an immediate and selective effect of the peptide strengthening neuronal systems of social memory.

Key words: oxytocin; social; face; recognition; memory; neuropeptide

\section{Introduction}

Across species, social recognition is the foundation on which all social relationships are built and maintained. The nonapeptide oxytocin is centrally involved in the regulation of basic social and reproductive behaviors, such as cohabitation, gestation, and breastfeeding, and in nonhuman mammals is crucial for social recognition (Carter, 1998, 2003; Ferguson et al., 2002; Winslow and Insel, 2004). In rodents, oxytocin enhances social recognition as indicated by decreased investigative behavior toward a conspecific rodent during a second encounter (Ferguson et al., 2002; Bielsky and Young, 2004). In oxytocin knock-out mice, social memory is impaired and can be fully restored by a single injection of oxytocin before an initial social encounter (Ferguson et al., 2002). Remarkably, oxytocin knock-out mice have no deficits in nonsocial memory (Ferguson et al., 2000), suggesting that oxytocin modulates only social but not nonsocial memory. The influence seems to be specific to encoding, because an injection of oxytocin before, but not after an initial social encounter restores social recognition in these mice (Ferguson et al., 2000).

In humans, the influence of oxytocin on social in comparison with nonsocial memory has not yet been investigated. However, there is emerging evidence that oxytocin facilitates social cognition and prosocial behavior also in humans (Heinrichs and Domes, 2008). Men treated with oxytocin performed better in inferring the affective state from the eye region of human faces (Domes et al., 2007a). Oxytocin also increases social behaviors like trust (Kosfeld et al., 2005; Baumgartner et al., 2008). In con-

Received Sept. 6, 2008; revised Nov. 12, 2008; accepted Nov. 24, 2008.

This work was supported by University of Zurich Young Investigator Research grants (Forschungskredit 2005 Forschungskredit 2006) (U.R.). U.R. was supported by Swiss National Science Foundation Postdoctoral Grant PBZH1118850 and by a grant from the Swiss Federal Institute of Sports. M.H. was supported by Swiss National Science Foundation Grant PP001-114788 and the Research Priority Program "Foundations of Human Social Behavior" at the University of Zurich. We thank Vera Dinkelacker for the face stimuli; Fabienne Marbacher, Manuel Schröter, Corina Winzer, Milenna van Dijk, and Claudia Zuccarella for their skillful assistance in data collection and analysis; and Elizabeth Phelps for helpful discussion on a previous version of this manuscript.

Correspondence should be addressed to Ulrike Rimmele, Department of Psychology, New York University, 6 Washington Place, Room 863, New York, NY 10003. E-mail: ur228@nyu.edu.

DOI:10.1523/JNEUROSCI.4260-08.2009

Copyright $\odot 2009$ Society for Neuroscience $\quad$ 0270-6474/09/290038-05\$15.00/0 trast, no consistent effects of oxytocin were found on learning of nonsocial stimuli (Fehm-Wolfsdorf and Born, 1991; Heinrichs et al., 2004). Here, we show that oxytocin selectively enhances memory encoding of faces in humans, but not of nonsocial stimuli.

\section{Materials and Methods}

Subjects. We studied 44 nonsmoking, healthy, heterosexual men without any psychiatric, neurological, and medical illness. Subjects were not on any medication and reported a normal sleep-wake cycle. The study was approved by the institutional review board of the University of Zurich. All subjects provided written informed consent and were paid for participation. Data from three subjects were excluded from analyses. One did not attend the second session, and two met criteria for a mental health disorder based on the Symptom-Checkliste SCL-90-R (Derogatis, 1983). Participants were instructed to abstain from beverages with caffeine or alcohol during experimental days and maintained a regular sleep-wake cycle the two nights before and during the study, with sleep between 10:00-11:30 P.M. and 7:00-8:30 A.M.

Social stimuli. A total of 120 grayscale Caucasian face stimuli (60 men; 60 women) was chosen from a pool of 780 faces that were selected from mainly four established databases: NimStim Face Stimulus Set (www. macbrain.org), Pictures of Facial Affect (Ekman and Friesen, 1971), International Affective Picture Set (Lang, 1999), Karolinska Directed Faces database (KDEF) (www.ki.SE/cns/news/AKDEF-e.html). All faces showed direct gaze and were presented in an elliptic mask on a black background. In a pilot study, a separate group of men $(n=50 ; 22.33 \pm$ 0.63 years) had rated the faces on a 7 point scale on valence (1, very negative; 7 , very positive) and arousal (1, not at all arousing; 7 , very arousing). A total of 120 of the 780 faces was then chosen ( 40 negative; 40 neutral; 40 positive) for the main study. A total of 84 faces ( 28 negative; 28 neutral; 28 positive) served as encoding material and 36 faces as distractors (12 negative; 12 neutral; 12 positive).

Nonsocial stimuli. A total of 120 grayscaled nonsocial stimuli (30 photographs of house fronts; 30 artificial objects that were photographs of art sculptures; 60 landscapes) served for assessment of nonsocial memory. Like the faces, they were presented in an elliptic mask on a black background. Twenty-one houses, 21 objects, and 42 landscapes were encoded, and 9 houses, 9 objects, and 18 landscapes served as distractors.

Design and procedure. A placebo-controlled, double-blind betweengroups design was used, with 22 men included in the oxytocin group (age, $22.59 \pm 0.57$ years) and 19 men $(22.53 \pm 0.52$ years $)$ in the placebo 
Table 1. Memory performance after oxytocin and placebo for faces and nonsocial stimuli

\begin{tabular}{|c|c|c|c|c|c|c|}
\hline & \multicolumn{2}{|c|}{ Oxytocin } & \multicolumn{2}{|c|}{ Placebo } & \multirow[b]{2}{*}{$t$} & \multirow[b]{2}{*}{$p$} \\
\hline & Mean & SEM & Mean & SEM & & \\
\hline \multicolumn{7}{|l|}{ Faces } \\
\hline Hit rate remember & 0.46 & 0.04 & 0.40 & 0.04 & 1.001 & 0.323 \\
\hline False alarm rate remember & 0.09 & 0.02 & 0.08 & 0.02 & 0.299 & 0.767 \\
\hline Hit rate know & 0.29 & 0.02 & 0.30 & 0.03 & -0.424 & 0.674 \\
\hline False alarm rate know & 0.20 & 0.02 & 0.26 & 0.03 & -2.030 & $0.049^{*}$ \\
\hline Recollection & 0.37 & 0.04 & 0.32 & 0.03 & 1.035 & 0.307 \\
\hline Familiarity & 0.33 & 0.03 & 0.22 & 0.03 & 2.504 & $0.017^{*}$ \\
\hline Overall recognition & 0.46 & 0.03 & 0.36 & 0.03 & 2.287 & $0.028^{*}$ \\
\hline \multicolumn{7}{|l|}{ Nonsocial } \\
\hline Hit rate remember & 0.56 & 0.04 & 0.53 & 0.04 & 0.658 & 0.515 \\
\hline False alarm rate remember & 0.11 & 0.02 & 0.10 & 0.02 & 0.561 & 0.578 \\
\hline Hit rate know & 0.22 & 0.03 & 0.25 & 0.02 & -0.822 & 0.416 \\
\hline False alarm rate know & 0.22 & 0.02 & 0.23 & 0.03 & -0.215 & 0.831 \\
\hline Recollection & 0.45 & 0.04 & 0.43 & 0.04 & 0.461 & 0.648 \\
\hline Familiarity & 0.24 & 0.03 & 0.27 & 0.02 & 0.859 & 0.396 \\
\hline Overall recognition & 0.44 & 0.04 & 0.44 & 0.03 & 0.035 & 0.973 \\
\hline
\end{tabular}

Overall recognition memory $=$ hit rate (remember + know $)-$ false alarm rate $($ remember + know). Recollection $=$ hit rate $($ remember $)-$ false alarm rate $($ remember $)$. Familiarity $=($ hit rate know/ $(1-$ hit rate remember $))-($ false alarm rate know/(1 - false alarm rate remember)).

The right two columns indicate results from pairwise statistical comparisons. ${ }^{*} p<0.05$, significant differences between oxytocin and placebo in memory measures for faces, but not for nonsocial stimuli.

group. Each volunteer in both groups participated in two experimental sessions taking place on 2 consecutive days. Sessions took place between 1:00 and 5:00 P.M. at the same time of day for each participant.

On day 1 , two groups of healthy young men were administered intranasally with oxytocin $(N=22)$ and placebo $(N=19)$. Recent research has shown that intranasal administration of neuropeptides, such as vasopressin, enables direct access of the peptide to the central nervous compartment (Born et al., 2002), thus providing a useful method for studying CNS effects of the neuropeptide oxytocin in humans, with no adverse side effects being reported (Heinrichs et al., 2003, 2004). Subjects received a single dose of 24 IU of oxytocin (Syntocinon Spray; Novartis; three puffs per nostril; each puff with 4 IU of oxytocin) intranasally or a placebo (containing all inactive ingredients except the neuropeptide). Forty minutes after substance administration, when central nervous oxytocin levels reached a plateau (Born et al., 2002), participants performed on a rating task for which the 84 faces and 84 nonsocial stimuli were presented in eight blocks containing 21 stimuli each, separated by breaks of $20 \mathrm{~s}$. Each picture was presented for $3.5 \mathrm{~s}$ in the middle of a 19 inch color monitor at a visual angle of $\sim 10^{\circ}$. Each picture appeared $2 \mathrm{~s}$ after presentation of the previous picture. During presentation, subjects had their heads fixated on a headrest. To assure proper encoding, the subject's task was to indicate how much he would like to approach the presented stimulus on a 7 point rating scale (1, not at all; 7 , very) (Adolphs et al., 1998) (see Fig. 1a). To keep encoding time constant for all stimuli, subjects were instructed to respond after the stimulus had disappeared from the monitor.

On day 2, $24 \mathrm{~h}$ later, a surprise recognition memory test was administered on the faces and nonsocial stimuli encoded the day before. The 84 encoded faces and the 84 encoded nonsocial stimuli were presented again, randomly intermixed with 36 new faces and 36 new nonsocial stimuli, respectively, in eight blocks of 30 stimuli. Subjects had to indicate whether they remembered (i.e., the presentation could be exactly recollected) or knew a stimulus (without recollecting the context of its presentation) or whether the picture was new (not seen at encoding) by pressing one of three response keys. Presentation time was $5 \mathrm{~s}$, with an interstimulus interval of $2 \mathrm{~s}$. Before this test, subjects had been trained to make remember/know judgments (Rajaram, 1993) with practice trials to verify that they fully understood the difference between a "remember" and "know" judgment. After recognition testing, subjects rated all stimuli on a 7 point scale with regard to valence (1, very negative; 7 , very positive) and arousal (1, not at all arousing; 7 , very arousing).

Overall recognition memory accuracy was assessed by subtracting the overall false alarm rate from the overall hit rate. Recollection and familiarity were estimated separately using remember and know responses. Recollection was assessed by subtracting the proportion of new items receiving a remember response (false alarms given a remember response) from the proportion of old items receiving a remember response (hits given a remember response). Familiarity was calculated as the probability for responding "know" to an item, given that the item was not given a remember response, corrected for false alarms [i.e. (hit rate know/(1 hit rate remember $)$ ) - (false alarm rate know/(1 - false alarm rate remember))] (see Table 1).

Attention, wakefulness, and mood. The influence of oxytocin on attention, wakefulness, and mood was assessed using the $\mathrm{d} 2$ letter cancellation test (Brickenkamp and Zillmer, 1998), the wakefulness scale of the Multidimensional Mood Questionnaire (Steyer et al., 1997), and the Positive and Negative Affect Scale (PANAS) (Watson et al., 1988). Measurements were taken before oxytocin or placebo administration, 1 min before encoding (wakefulness; mood) and 1-5 min after encoding (wakefulness; mood; attention). At recognition testing, the same questionnaires were given before recognition testing and after the rating of the stimuli. Al questionnaires have been broadly used and shown satisfactory internal consistency and validity.

Statistical analysis. Statistical analyses relied on ANOVA, which included a group factor treatment (oxytocin vs placebo) and, for analysis of recognition performance, a repeated-measures factor stimulus category (faces vs nonsocial). Additionally, ANOVAs for repeated measures investigated whether gender or valence of the faces contributed to the influence of oxytocin on recognition memory. Significant ANOVA main effects or interactions were specified by sub-ANOVAs and independent sample $t$ tests. To explore whether arousal (as rated after recognition testing) modulated the influence of oxytocin on recognition memory, the individual average arousal ratings were introduced as covariates. Where appropriate, degrees of freedom were adjusted according to the Greenhouse-Geisser procedure. Effects on measures of attention, wakefulness, and mood were likewise assessed by ANOVA. To investigate whether subjects were aware of their drug condition, a $\chi^{2}$ test was used. Data are presented as mean \pm SEM. All tests were two-sided and the level of significance was set at $p<0.05$. All statistical analyses were performed using SPSS 16 (SPSS).

\section{Results}

\section{Rating of approachability during stimulus encoding}

The oxytocin and placebo groups did not differ in their approachability ratings for faces (oxytocin, $3.56 \pm 0.12$; placebo, $3.64 \pm 0.14$ ) and nonsocial stimuli (oxytocin, $4.59 \pm 0.12$; placebo, $4.34 \pm 0.12$ ) during the encoding phase (values of $p>$ $0.13)$. In addition, no significant difference in rated approachability was observed between the treatment groups for faces sub- 
divided into negative (oxytocin, $2.46 \pm$ 0.17; placebo, $2.47 \pm 0.14$ ), neutral (oxytocin, $3.90 \pm 0.12$; placebo, $4.16 \pm 0.16$ ), and positive (oxytocin, $4.31 \pm 0.17$; placebo, $4.29 \pm 0.22$ ) valence categories (all values of $p>0.18$ ).

\section{Recognition of faces versus nonsocial stimuli}

Oxytocin differentially influenced overall recognition accuracy (hit rate - false alarm rate collapsed across remember and know responses) of social versus nonsocial stimuli (treatment by category interaction; $\left.F_{(1,39)}=4.90 ; p<0.05\right)$ (Fig. $1 b$; Table 1$)$. Recognition accuracy for faces was superior in the oxytocin $(0.46 \pm 0.03)$ compared with the placebo group $(0.36 \pm$ $\left.0.027 ; t_{(39)}=2.29 ; p<0.05\right)$, whereas recognition accuracy for the nonsocial stimuli was comparable in both groups $(p>$ 0.97).

Analyses of recollection and familiarity revealed a more fine-grained picture of the oxytocin effect. Whereas oxytocin did not affect recollection (all values of $p>0.42$ ) (Fig. $1 c$; Table 1), the peptide strikingly affected familiarity judgments, depending on whether the stimulus was a face or not (treatment by category interaction; $F_{(1,39)}=14.32 ; p<0.001$ ) (Fig. 1d; Table 1). Familiarity judgments of the faces were superior in the oxytocin $(0.33 \pm 0.03)$ than in the placebo group $\left(0.22 \pm 0.028 ; t_{(39)}=2.50 ; p<0.05\right)$. Specifically, subjects in the oxytocin group were less likely to give a know response to a new face $(0.198 \pm 0.02)$ than subjects in the placebo group $(0.26 \pm$ $\left.0.03 ; t_{(39)}=2.03 ; p<0.05\right)$. For nonsocial stimuli, familiarity scores $(p>0.39)$ as well as number of false know responses $(p>$ 0.80 ) were comparable between the treatment groups.

\section{Influence of gender and valence on face recognition}

To explore whether the improved memory for faces after oxytocin was dependent on the gender of the face or its rated valence, ANOVAs were run including either an additional gender factor (male; female) or a valence factor (negative; neutral; positive).

Valence rated at the time of recognition testing, as expected, confirmed the differences between faces grouped into the negative $(2.21 \pm 0.09)$, neutral $(3.77 \pm 0.09)$, and positive face categories in all subjects $(4.19 \pm 0.12$; all values of $p<0.001)$. The oxytocin and placebo groups did not differ in their valence ratings (all values of $p>0.49$ ). Analysis of overall recognition accuracy, apart from confirming the improving effect of oxytocin $\left(t_{(39)}=2.29 ; p<0.05\right)$, revealed a main effect of valence $\left(F_{(2,78)}=\right.$ $33.2 ; p<0.001)$, indicating that negative $(0.17 \pm 0.01)$ and positive faces $(0.16 \pm 0.01)$ were generally recognized better than neutral faces $(0.09 \pm 0.01$; values of $p<0.001)$. However, this effect was not modulated by oxytocin $(p>0.82$ for respective treatment by valence interaction). Analysis of familiarity scores, which were found to be most sensitive to the influence of oxytocin on face memories in the main analyses, revealed a similar picture. Familiarity differed between faces of different valence (main effect of valence with higher scores for negative and positive than neutral faces; $\left.F_{(2,78)}=6.56 ; p<0.01\right)$. However, this effect again did not depend on the type of treatment $(p>0.89$ for

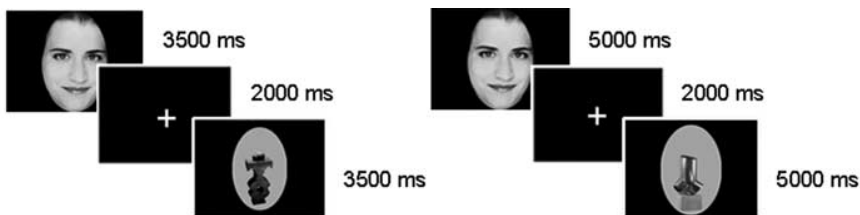

How much would you like to approach this person / object?

Remember, know, new?

$\rightarrow$ Encoding

$\leftarrow 24$ hours

Surprise recognition test

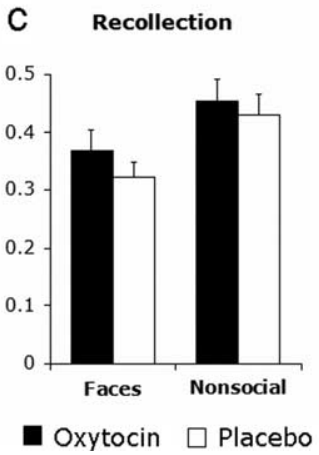

Oxytocin $\square$ Placebo

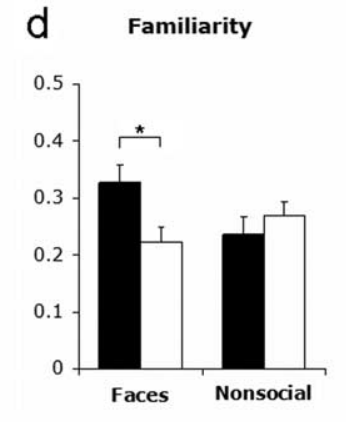

social (abstract objects) stimuli. $\boldsymbol{b}, \boldsymbol{d}, 0 x y$ tocin enhances overall recognition memory accuracy $(\boldsymbol{b})\left(F_{(1,39)}=4.90 ; p<0.05\right.$; treatment by category interaction), and familiarity (d) for faces, but not for nonsocial stimuli $\left(F_{(1,39)}=14.32 ; p<0.001\right.$; treatment by category interaction). The oxytocin group showed a significant higher familiarity for faces compared with the placebo group $\left(t_{(39)}=2.50 ; p=0.017\right)$. c, Recollection of faces was not influenced by oxytocin. ${ }^{*} p<0.05$ for pairwise comparisons. Error bars indicate SEM.

treatment by valence). Overall, these analyses did not reveal valence as a factor of any importance for the improving influence of oxytocin on face recognition.

Gender of the face was likewise excluded as modulator of the effects of the peptide on face recognition. There was no significant effect of gender on overall recognition accuracy (all values of $p>0.78$ ). Regarding familiarity scores, male faces were more familiar than female faces (male, $0.16 \pm 0.01$; female, $0.12 \pm 0.02$; main effect of gender; $\left.F_{(1,39)}=5.05 ; p<0.05\right)$. The false alarm rate for know responses was also lower for male $(0.10 \pm 0.01)$ than female faces $(0.13 \pm 0.01)\left(F_{(1,39)}=4.93 ; p<0.05\right)$. However, these effects of gender on familiarity and false alarms were again comparable in the placebo and oxytocin groups (values of $p>0.28$ for treatment by gender).

\section{Influence of arousal on recognition performance}

Because previous studies indicated strong influences of arousal ratings of pictures on encoding of memories (McGaugh and Roozendaal, 2002; LaBar and Cabeza, 2006), in supplementary analyses we controlled for this possible influence by entering arousal (as rated after recognition) as covariate into ANOVAs of memory performance. None of these analyses revealed any significance for the arousal covariate (all values of $p>0.21$ ), but they all confirmed the main effects of oxytocin reported for the original analyses.

\section{Attention, wakefulness, mood, and substance awareness} Attention, as assessed by letter cancellation performance, increased from before substance administration (oxytocin, $397.2 \pm$ 29.0; placebo, $390.78 \pm 33.1)$ to the time after the period of stimulus encoding (oxytocin, $459.5 \pm 36.4$; placebo, $424.1 \pm$ 33.7) $\left(F_{(1,39)}=20.65 ; p<0.001\right)$, but did not differ between groups (all values of $p>0.17$ ). Wakefulness did not change throughout the encoding session and also was not influenced by 
oxytocin (all values of $p>0.19$ ). Negative affect, as assessed by the PANAS, decreased across the encoding session $\left(F_{(1,39)}=8.16\right.$; $p<0.01)$, but the measure did not differ between oxytocin and placebo groups (all values of $p>0.27$ ). At recognition testing, again no differences between treatment groups were observed in all psychological measures (all values of $p>0.63$ ). When asked in the end of the second session, subjects were unable to correctly identify whether they had received an active agent or placebo $\left(\chi^{2}\right.$ test; $p>0.25)$.

\section{Discussion}

This is the first study to show that oxytocin improves recognition for faces, but not for nonsocial stimuli in humans. Our data indicate that a single dose of intranasally administered oxytocin 40 min before encoding causes a substantial improvement in the ability to recognize faces a day later, while leaving entirely unaffected the recognition of nonsocial stimuli. Our finding is consistent with previous observations suggesting a globally enhancing effect of oxytocin on processing of face stimuli (Guastella et al., 2008a,b; Savaskan et al., 2008). Most importantly, this result concurs with rodent studies that show oxytocin to be selectively essential for the establishment of memory of a conspecific, but not for learning of nonsocial information (Ferguson et al., 2000, 2002).

More specifically, the enhancing effect of oxytocin on recognition of faces versus nonsocial stimuli emerged for familiarity judgments, whereas recollection remained unaffected. The peptide strikingly improved familiarity judgments for faces, but not for nonsocial stimuli. This pattern shows that the advantage in social recognition after oxytocin expresses itself in a greater familiarity of previously encountered faces. Specifically, oxytocin lowered the detection threshold for faces. Indeed, the raw measure mainly affected was the false alarm rate for know responses, which was decreased after oxytocin administration, thereby distinctly improving the signal-to-noise ratio for discriminating new faces from old ones. Remarkably, recollection measures of face memory remained unaffected by oxytocin. Recollection reflects the conscious effortful retrieval of qualitative information of a study event, whereas familiarity judgments are based on a direct sensing of the memory strength (Yonelinas, 2002). Hence, the specific effect of oxytocin on familiarity of faces likely reflects an immediate strengthening of neuronal circuitry selectively representing social memories.

Additional analyses did not reveal any considerable influence of gender and valence of the face, or arousal on memory performance (all values of $p>0.21$ ). Thus, the enhancing effect of oxytocin on recognition and familiarity of faces is independent of whether the face is that of a man or a woman, and whether the face is experienced as negative, neutral, or positive, indicating that the social nature of the stimulus per se is relevant for the memory effect of the peptide regardless of its phenotypic appearance (Savaskan et al., 2008). Also, there was no difference between the oxytocin and placebo groups in any measure of attention, alertness, and mood at encoding or at recognition testing, which excludes that differences in recognition were confounded by effects of the peptide on these nonspecific functions.

Together, our data indicate that oxytocin in humans immediately strengthens the capability to correctly recognize and discriminate faces. The identification of an evolutionarily highly preserved hormone accounting for improved recognition of conspecifics not only in rodents, but also in humans, points to a similar mechanism of social recognition across species.

In rodents, oxytocin is essential in the medial amygdala for establishing a social memory (Ferguson et al., 2002). Olfactory cues of a conspecific are conveyed via the main and accessory olfactory pathways to the medial amygdala where oxytocin acts to modulate encoding of the memory for the initial social encounter (Ferguson et al., 2002). The medial amygdala projects to the bed nucleus of the stria terminalis and via the lateral septum to the hippocampus, which is most crucial for storage and retrieval of many types of memories. After a social exposure, oxytocin knock-out mice show hypoactivation of the medial amygdala and several downstream projections of this nucleus (Ferguson et al., 2001). Interestingly, these mice simultaneously show a massive hyperactivation of other brain areas including the hippocampus and somatosensory cortex, which possibly reflects the recruitment of alternative pathways for processing social cues. Oxytocin administered into the medial amygdala of knock-out mice before an initial social encounter fully restores social recognition (Ferguson et al., 2001). Conversely, an oxytocin receptor antagonist administered to the medial amygdala of wild-type mice impairs social recognition. These findings show that the activation of oxytocin receptors in the medial amygdala is both necessary and sufficient for the successful formation of a social memory in mice.

In humans, faces constitute the primary social cue. The processing of faces relative to other visual stimuli recruits a distributed neural system in the human brain (Haxby et al., 2000). In particular, among other regions, numerous neuroimaging studies identified the amygdala, the superior temporal sulcus (STS), and a region in the fusiform gyrus, the fusiform face area (FFA), to be critically involved in face processing (Sergent et al., 1992; Haxby et al., 1994; Kanwisher et al., 1997; McCarthy et al., 1997) (for review, see Haxby et al., 2000; Adolphs, 2002; Vuilleumier, 2007). It has been proposed that the fusiform face area processes invariant aspects of faces and thus contributes to face identity perception, whereas the STS and the amygdala are more important for the processing of variable aspects of faces, such as eye gaze and emotional expression. However, these regions do not act separately from each other, but rather interact with each other, in particular the amygdala and the FFA (Vuilleumier, 2007).

The neuronal mechanisms mediating the effects of oxytocin on human face memories cannot be inferred from the present data. Expression of oxytocin receptors has been revealed in various brain regions, particularly in the amygdala and hippocampus (Insel and Shapiro, 1992; Gimpl and Fahrenholz, 2001; Landgraf and Neumann, 2004; Huber et al., 2005), but also in different regions of the neocortex (Insel et al., 1991; Gimpl and Fahrenholz, 2001). Recent functional magnetic resonance imaging studies indicated a specific modulation of amygdalar activity during face processing after administration of oxytocin (Kirsch et al., 2005; Domes et al., 2007b; Petrovic et al., 2008). Of note, one of these studies revealed oxytocin induced blood oxygen leveldependent signal changes also in the fusiform gyrus (Petrovic et al., 2008). Both amygdala and fusiform gyrus are involved in the acquisition of familiarity for faces (Kosaka et al., 2003). In previous experiments using the same face presentation as well as dose and timing of oxytocin as in the present study, we found that oxytocin administered before face encoding reduces activity in the amygdala (Domes et al., 2007b). This effect, like the enhancing effect of oxytocin on face familiarity in the present study, was independent on the valence or arousal value of the faces. Given that the amygdala contributes to emotion perception in faces, whereas face identity is mediated via the FFA (Adolphs, 2002; Vuilleumier, 2007), the independence on valence and arousal so consistently observed for the effects of oxytocin suggests a primary role for the FFA (rather than amygdala) in mediating the 
enhanced facial familiarity after oxytocin, revealed in here. The focus of the effect of the peptide on familiarity rather than recollection of faces likewise suggests that oxytocin predominantly acts at the neocortical level of the face encoding circuitry. In combination, our observations argue for the view that the enhanced familiarity judgments for faces encoded under the influence of oxytocin involve both an action on the amygdala as well as the fusiform face area, with perhaps a leading role for the influence on the fusiform gyrus.

In summary, the findings of our study show a crucial function of oxytocin in the early processing of the most basic class of social stimuli (i.e., faces). Similar to animal studies, we find that oxytocin strengthens the encoding of conspecifics and is essential for the identification and recognition of individual conspecifics. Social recognition is an essential prerequisite of more complex social behaviors. Many animal and a few human studies have shown that oxytocin is involved in the regulation of complex social behaviors, such as trust, pair bonding, or parental care. In the light of our basic findings, oxytocin appears to provide an effective approach for selectively influencing and ameliorating the foundation of basic social competences in humans, especially in disease conditions.

\section{References}

Adolphs R (2002) Recognizing emotion from facial expressions: psychological and neurological mechanisms. Behav Cogn Neurosci Rev 1:21-62.

Adolphs R, Tranel D, Damasio AR (1998) The human amygdala in social judgment. Nature 393:470-474.

Baumgartner T, Heinrichs M, Vonlanthen A, Fischbacher U, Fehr E (2008) Oxytocin shapes the neural circuitry of trust and trust adaptation in humans. Neuron 58:639-650.

Bielsky IF, Young LJ (2004) Oxytocin, vasopressin, and social recognition in mammals. Peptides 25:1565-1574.

Born J, Lange T, Kern W, McGregor GP, Bickel U, Fehm HL (2002) Sniffing neuropeptides: a transnasal approach to the human brain. Nat Neurosci 5:514-516.

Brickenkamp R, Zillmer E (1998) The d2 Test of Attention. Seattle: Hogrefe and Huber.

Carter CS (1998) Neuroendocrine perspectives on social attachment and love. Psychoneuroendocrinology 23:779-818.

Carter CS (2003) Developmental consequences of oxytocin. Physiol Behav 79:383-397.

Derogatis L (1983) SCL-90-R administration, scoring and procedures manual, II. Towson, MD: Clinical Psychometric Research.

Domes G, Heinrichs M, Michel A, Berger C, Herpertz SC (2007a) Oxytocin improves "mind-reading" in humans. Biol Psychiatry 61:731-733.

Domes G, Heinrichs M, Gläscher J, Büchel C, Braus DF, Herpertz SC (2007b) Oxytocin attenuates amygdala responses to emotional faces regardless of valence. Biol Psychiatry 62:1187-1190.

Ekman P, Friesen WV (1971) Constants across cultures in the face and emotion. J Pers Soc Psychol 17:124-129.

Fehm-Wolfsdorf G, Born J (1991) Behavioral effects of neurohypophyseal peptides in healthy volunteers: 10 years of research. Peptides 12:1399-1406.

Ferguson JN, Young LJ, Hearn EF, Matzuk MM, Insel TR, Winslow JT (2000) Social amnesia in mice lacking the oxytocin gene. Nat Genet 25:284-288.

Ferguson JN, Aldag JM, Insel TR, Young LJ (2001) Oxytocin in the medial amygdala is essential for social recognition in the mouse. J Neurosci 21:8278-8285.

Ferguson JN, Young LJ, Insel TR (2002) The neuroendocrine basis of social recognition. Front Neuroendocrinol 23:200-224.

Gimpl G, Fahrenholz F (2001) The oxytocin receptor system: structure, function, and regulation. Physiol Rev 81:629-683.

Guastella AJ, Mitchell PB, Dadds MR (2008a) Oxytocin increases gaze to the eye region of human faces. Biol Psychiatry 63:3-5.

Guastella AJ, Mitchell PB, Mathews F (2008b) Oxytocin enhances the en- coding of positive social memories in humans. Biol Psychiatry 64:256-258.

Haxby JV, Horwitz B, Ungerleider LG, Maisog JM, Pietrini P, Grady CL (1994) The functional organization of human extrastriate cortex: a PETrCBF study of selective attention to faces and locations. J Neurosci 14:6336-6353.

Haxby JV, Hoffman EA, Gobbini MI (2000) The distributed human neural system for face perception. Trends Cogn Sci 4:223-233.

Heinrichs M, Domes G (2008) Neuropeptides and social behavior: effects of oxytocin and vasopressin in humans. Prog Brain Res 170:337-350.

Heinrichs M, Baumgartner T, Kirschbaum C, Ehlert U (2003) Social support and oxytocin interact to suppress cortisol and subjective responses to psychosocial stress. Biol Psychiatry 54:1389-1398.

Heinrichs M, Meinlschmidt G, Wippich W, Ehlert U, Hellhammer DH (2004) Selective amnesic effects of oxytocin on human memory. Physiol Behav 83:31-38.

Huber D, Veinante P, Stoop R (2005) Vasopressin and oxytocin excite distinct neuronal populations in the central amygdala. Science 308:245-248.

Insel TR, Shapiro LE (1992) Oxytocin receptor distribution reflects social organization in monogamous and polygamous voles. Proc Natl Acad Sci U S A 89:5981-5985.

Insel TR, Gelhard R, Shapiro LE (1991) The comparative distribution of forebrain receptors for neurohypophyseal peptides in monogamous and polygamous mice. Neuroscience 43:623-630.

Kanwisher N, McDermott J, Chun MM (1997) The fusiform face area: a module in human extrastriate cortex specialized for face perception. J Neurosci 17:4302-4311.

Kirsch P, Esslinger C, Chen Q, Mier D, Lis S, Siddhanti S, Gruppe H, Mattay VS, Gallhofer B, Meyer-Lindenberg A (2005) Oxytocin modulates neural circuitry for social cognition and fear in humans. J Neurosci 25:11489-11493.

Kosaka H, Omori M, Iidaka T, Murata T, Shimoyama T, Okada T, Sadato N, Yonekura Y, Wada Y (2003) Neural substrates participating in acquisition of facial familiarity: an fMRI study. Neuroimage 20:1734-1742.

Kosfeld M, Heinrichs M, Zak PJ, Fischbacher U, Fehr E (2005) Oxytocin increases trust in humans. Nature 435:673-676.

LaBar KS, Cabeza R (2006) Cognitive neuroscience of emotional memory. Nat Rev Neurosci 7:54-64.

Landgraf R, Neumann ID (2004) Vasopressin and oxytocin release within the brain: a dynamic concept of multiple and variable modes of neuropeptide communication. Front Neuroendocrinol 25:150-176.

Lang P (1999) International Affective Picture System (IAPS): instruction manual and affective ratings. Technical Report A-4, The Center for Research in Psychophysiology. Gainesville, FL: University of Florida.

McCarthy G, Puce A, Gore JC, Allison T (1997) Face-specific processing in the human fusiform gyrus. J Cogn Neurosci 9:605-610.

McGaugh JL, Roozendaal B (2002) Role of adrenal stress hormones in forming lasting memories in the brain. Curr Opin Neurobiol 12:205-210.

Petrovic P, Kalisch R, Singer T, Dolan RJ (2008) Oxytocin attenuates affective evaluations of conditioned faces and amygdala activity. J Neurosci 28:6607-6615.

Rajaram S (1993) Remembering and knowing: two means of access to the personal past. Mem Cognit 21:89-102.

Savaskan E, Ehrhardt R, Schulz A, Walter M, Schächinger H (2008) Postlearning intranasal oxytocin modulates human memory for facial identity. Psychoneuroendocrinology 33:368-374.

Sergent J, Ohta S, MacDonald B (1992) Functional neuroanatomy of face and object processing. A positron emission tomography study. Brain 115:15-36.

Steyer R, Schwenkmezger P, Notz P, Eid M (1997) Der Mehrdimensionale Befindlichkeitsbogen (MDBF). Göttingen: Hogrefe.

Vuilleumier P (2007) Neural representation of faces in human visual cortex: the roles of attention, emotion, and viewpoint. Tokyo, Berlin, Heidelberg, New York: N. Osaka and I. Rentschler and I. Biederman.

Watson D, Clark LA, Tellegen A (1988) Development and validation of brief measures of positive and negative affect: the PANAS scales. J Pers Soc Psychol 54:1063-1070.

Winslow JT, Insel TR (2004) Neuroendocrine basis of social recognition. Curr Opin Neurobiol 14:248-253.

Yonelinas AP (2002) The nature of recollection and familiarity: a review of 30 years of research. J Mem Lang 46:441-517. 